\title{
PERFIL NUTRICIONAL DE PACIENTES IDOSOS COM COVID-19: UMA REVISÃO INTEGRATIVA
}

Raquel Seibel; Pontifícia Universidade Católica do Rio Grande do Sul (PUCRS); raquelseibel27@gmail.com; Jamile Ceolin; Pontifícia Universidade Católica do Rio Grande do Sul (PUCRS); jamileceolin@yahoo.com.br

\section{RESUMO}

Introdução: A pandemia da COVID-19 representa um desafio para os sistemas de saúde mundialmente. Os idosos, que apresentam múltiplas comorbidades crônicas, são mais suscetíveis ao COVID-19 e a piora do estado nutricional, principalmente em pacientes hospitalizados. Objetivo: Avaliar o perfil nutricional e desfechos clínicos de pacientes idosos com COVID-19. Métodos: Foi realizada uma revisão integrativa com ensaios clínicos, através de uma busca na base de dados Medline (via Pubmed). A sequência da busca utilizada foi: [(Coronavirus infections) AND (Nutritional Status)]. Foram encontrados 54 artigos. Após a leitura dos estudos foram excluídos 42 artigos por não avaliarem idosos, 4 por não serem com humanos, 3 por serem revisão, 1 por não especificar idade, 1 por não abordar o estado nutricional e 1 por ser em chinês. Resultados: Foram incluídos 2 artigos, um com delineamento transversal e outro retrospectivo, totalizando 323 pacientes hospitalizados e com diagnóstico de COVID-19. A amostra era predominantemente feminina, e a média de idade foi de 70,05 7,3 anos. O estado nutricional foi avaliado através da Mini Avaliação Nutricional, Nutrition Risk Screening 2002, Malnutrition Universal Screening Tool e Índice de Risco Nutricional. Nos idosos com COVID-19, houve alta prevalência de desnutrição e risco nutricional, associado com maior tempo de internação, elevadas despesas hospitalares, pior gravidade da doença e maior alteração de peso do que o grupo com estado nutricional normal. Conclusão: Os resultados sugerem que idosos com COVID-19, em risco nutricional, podem apresentar desfechos clínicos piores do que indivíduos com estado nutricional adequado, entretanto mais estudos são necessários.

Palavras-chave: Estado nutricional; Idoso; Infecções por Coronavírus; Hospitalização. 